\title{
Translation of a circulating microRNA signature of melanoma into a solid tissue assay with the potential to improve diagnostic precision and reproducibility
}

\section{Ryan Van Laar ( $\square$ vanlaar@geneseq.com.au )}

Geneseq Biosciences https://orcid.org/0000-0003-2828-4175

\section{Samuel King}

Australian Clinical Labs

Richard McCoy

Australian Clinical Labs

Mirette Saad

Australian Clinical Labs

\section{Sian Fereday}

Geneseq Biosciences

Ingrid Winship

Geneseq Biosciences

\section{Catherine Uzzell}

Australian Clinical Labs

\section{Anthony Landgren}

Australian Clinical Labs

\section{Research Article}

Keywords: Diagnostic Accuracy, Reproducibility, Solid-Tissue Biomarker

Posted Date: May 19th, 2021

DOl: https://doi.org/10.21203/rs.3.rs-77357/v4

License: (9) This work is licensed under a Creative Commons Attribution 4.0 International License. Read Full License 


\section{Abstract}

Background: Successful treatment of cutaneous melanoma depends on early and accurate diagnosis of clinically suspicious melanocytic skin lesions. Multiple international studies have described the challenge of providing accurate and reproducible histopathological assessments of melanocytic lesions, highlighting the need for new diagnostic tools including disease-specific biomarkers. Previously, a 38microRNA signature ("Mel38") was identified in melanoma patient plasma and validated as a novel biomarker. In this study, Mel38 expression in solid tissue biopsies representing the benign naevi to metastatic melanoma spectrum is examined.

Methods: Nanostring digital gene expression assessment of the Mel38 signature was performed on 308 formalin fixed paraffin embedded biopsies of naevi, melanoma in-situ and invasive melanoma. Genomic data were interrogated using hierarchical clustering, univariate, and multivariate statistical approaches. Classification scores computed from the Mel38 signature were analysed for their association with demographic data and histopathology results, including MPATH-DX class, AJCC disease stage and tissue subtype.

Results: The Mel38 score can stratify higher-risk melanomas (MPATH-Dx Class V or more advanced) from lower-risk skin lesions (Class I-IV) with an area-under-the curve of $0.97(P<0.001)$. The genomic score ranges from 0 to 10 and is positively correlated with melanoma progression, with an intraclass correlation coefficient of 0.85 with stage 0 to IV disease. Using an optimised classification threshold of $\geq 2.7$ accurately identifies higher-risk melanomas, associated with poorer outcomes and more intensive suggested clinical actions with $89 \%$ sensitivity and $94 \%$ specificity. Multivariate analysis showed the score to be a significant predictor of malignancy, independent of technical and clinical covariates. Application of the Mel38 signature to spitz naevi reveal an intra-subtype profile, with elements of the profile in common to both naevi and melanoma.

Conclusion: Melanoma-specific circulating microRNAs maintain their association with malignancy when measured in the hypothesized tissue of origin. The Mel38 signature is an accurate and reproducible metric of melanoma status, based on changes in microRNA expression that occur as the disease develops and spreads. Inclusion of the Mel38 score into routine practice would provide physicians with previously unavailable, personalised genomic information about their patient's skin lesions. Combining molecular biomarker data with conventional histopathology data may improve diagnostic accuracy, healthcare resource utilisation, and patient outcomes.

\section{Background}

Over the past two decades, the annual incidence of melanoma has increased by $4-6 \%$ in fair-skinned populations of Northern Europe, North America, New Zealand and Australia[1]. Australia leads the world in melanoma incidence, with two out of three people diagnosed with some form of skin cancer by the age of 70[2]. Unfortunately, public awareness campaigns and advances in diagnosis and therapeutics have 
not translated to prolonged improvements in the age-standardised mortality rates for this cancer type in many countries[3-6].

Currently, melanocytic lesions suspected of being malignant are diagnosed almost exclusively using visual methods, including dermoscopy, followed by biopsy and histopathologic examination. Previous advances in screening and diagnosis have increased the incidence of early-stage melanoma diagnoses worldwide but have not reduced overall mortality rates[3]. Over the past decade, new treatments including targeted therapies and immunotherapy have shown promise in reducing mortality. However, these treatments are approved for late-stage melanoma patients only, can cost in excess of US\$100,000 per patient per year and their long-term benefit is still being studied[7, 8].

The MPATH-Dx structure for describing melanocytic lesions is increasingly adopted by pathologists as a method to improve diagnostic consistency and communication between healthcare providers $[9,10]$. Despite this and other improvements in related areas such as dermoscopy, multiple studies into the accuracy and reproducibility of melanoma diagnosis by histopathology alone show that it is not adequately accurate or reproducible[11-18] (see Table 1). Elmore et al showed that $28 \%$ of MPATH-Dx Class $\vee$ melanomas, i.e., those requiring the widest excision margins and consideration of lymph node screening and adjuvant therapy, were significantly under-diagnosed. Reproducibility of Class $V$ diagnoses was also lacking, with $17 \%$ of Class $V$ lesions examined receiving a lower classification when the same diagnostic slides were re-analysed by the same pathologist at a different time point. There is consensus among experts that the introduction of standardised pathology terminology and new molecular tests are needed to improve diagnostic accuracy, patient outcomes and resource use efficiency[4, 12, 13, 19-21].

Genomic profiling of microRNAs is a precise molecular technique that can be performed on many tissue types, including blood, urine, tears, and solid tissue (Figure 1). MicroRNAs are post-transcriptional regulators of gene expression which have tissue- and disease-specific patterns of expression[22]. Their role in melanoma oncogenesis and progression is well documented, including their crucial role in formation of the dermal tumour niche and activation of cancer associated fibroblasts through exosomal secretion. Studies have shown that microRNAs can have either pro- or anti oncogenic actions, resulting in increasing or decreasing levels of their expression as dermal cells develop malignant properties, interact with their microenvironment and invade nearby or distant tissues[23].

Previously, a signature of 38 circulating microRNAs ('Mel38') was identified by genomic profiling of plasma from individuals with stage I-IV melanoma and comparison with data from non-melanoma controls. The biological functions of the Mel38 microRNAs include regulators of angiogenesis and inflammation $(n=2)$, invasion and metastasis $(n=14)$, immune response and treatment resistance $(n=11)$ and tumour suppression and oncogene activation $(n=8)[24,25]$. The protein-coding mRNAs identified as those regulated by Mel38 overlap significantly with the MAPK signalling pathway $\left(P=1.3 \times 10^{-11}\right)$ [26]. Modulation of this pathway is responsible for therapeutically-targetable BRAF and NRAS mutations in melanoma cells[27]. 
To express the information represented by the Mel38 signature in an easily interpretable and patientpersonalised form, a gene weighting algorithm is applied to the Nanostring-quantified microRNA counts to generate a classification score (i.e., Mel38 score). Mel38 scores ranges from 0 to 10 and are positively associated with increasing levels of melanoma malignancy, from benign naevi to metastatic disease. In multiple validation cohorts comprised of varied specimen types, the Mel38 score has shown robust and statistically significant associations with melanoma status, treatment response and prognosis[24-26].

In this study we perform Mel38 microRNA profiling of 308 clinically annotated, formalin fixed paraffin embedded melanocytic skin lesions, representing the most common specimen and histological subtypes analysed in skin pathology practices. The clinical and technical accuracy of the Mel38 signature is evaluated and contrasted with equivalent metrics for conventional diagnostic approaches to demonstrate the potential benefit of including genomic profiling in the diagnostic workup of melanocytic lesions.

\section{Patients And Methods}

\section{Sample population}

This retrospective study was performed using melanocytic lesions that were submitted for diagnostic assessment (Australian Clinical Labs) as part of normal health care over the prior five years. Specimens were selected to represent the progression from benign naevi through to advanced invasive adult melanoma (18 years and older), including multiple common histological subtypes and disease stages (AJCC $8^{\text {th }}$ Ed[28]). An additional 14 samples of spitz naevi from patients younger than 18 at the time of diagnosis were also included.

Each specimen was reviewed by two or more experienced dermatopathologists, with the majority diagnosis recorded as the result. The study was approved by Australian Clinical Labs internal medical advisory board and satisfies criteria for use of human tissue by diagnostic pathology companies as outlined by the Australian Government's National Health and Medical Research Council [29]. A study sample size of $\geq 300$ specimens was set based on the methods described by Hajian-Tilak, assuming an AUC of 0.78 or higher with $80 \%$ power and $95 \%$ confidence[30].

\section{RNA extraction and genomic analysis}

A minimum of two unstained slides containing a total of $20 \mu \mathrm{m}$ of tissue with $20 \%$ or greater cellularity was obtained from each specimen. The reviewing pathologist indicated the area of interest for each specimen using a Hematoxylin and eosin stained slide which then guided the macro-dissected process on the unstained slides. Total RNA was extracted from deparaffinated tissue using the miRNeasy FFPE kit (QIAGEN Cat No./ID: 217504) according to manufacturer guidelines and quantified using a Qubit 2.0 fluorometer (Invitrogen, USA). MicroRNA profiling was performed using the Nanostring nCounter Human v3 miRNA Panel with the nCounter SPRINT or nCounter MAX platform (Nanostring Inc, Seattle USA), according to the manufacturer's guidelines. 


\section{Data processing and statistical analysis}

Log2 scaled raw data were adjusted for technical variation by positive control scaling and 'top 100' normalisation using the NanoStringNorm R library [31]. Normalised data were then examined for quality by comparing 10 quality metrics with predetermined internal standards, including assessments of positive and negative controls, ligation efficiency controls, global mean and standard deviation and the degree of normalisation applied. A classification score ('Mel38 score') was calculated for each specimen that passed the data quality control analysis, using the support vector machine (SVM) gene weighting algorithm, described previously[25]. The SVM microRNA expression weights used to compute a Mel38 score were updated for differences between plasma and FFPE derived data by performing a partial retraining using representative subset of nevi $(n=12)$ and invasive melanoma samples $(n=12)$.

All data normalisation, quality control and Mel38-FFPE score calculations were performed using customised R scripts and the results stored in a relational database. Statistical analysis and visualisation were performed using Microsoft Excel and MedCalc [32, 33]. All P-values calculated are two-sided and when $<0.05$ were deemed to be statistically significant. Receiver operator curve (ROC) analysis was used to assess the sensitivity and specificity of the Mel38-FFPE score and to determine the optimal score threshold to classify specimens as either high or low risk of disease progression and a potentially poorer outcome[34]. Binary logistic regression was used visualise the score as a continuous predictor of malignancy.

\section{Results}

\section{Genomic profiling of melanocytic lesions representing the spectrum of benign melanocytic lesion to metastatic melanoma.}

The Mel38 signature was examined using total RNA from 308 FFPE tissue specimens with a melanocytic cellular content of $10 \%$ or greater. Patient and specimen details are summarised in Table 1. To visualise the relationship between the 38 microRNAs and specimen details, two-dimensional hierarchical clustering of the complete cohort was performed (Figure 2). This resulted in an ordering of samples corresponding to an increasing degree of cancer progression (left to right). The gradual transition in levels of relative up and down microRNA expression reflects the continuum of naevi to invasive melanoma progression, as seen in clinical practice.

Notably, two microRNAs (hsa-mir-205 and hsa-mir-497) appear to have similarly low expression in the benign naevi and invasive/metastatic melanomas, but high expression in the early-stage invasive melanomas. These microRNAs may therefore have a specific role in aiding a melanoma cell to develop early invasive characteristics and may warrant further investigation. 


\section{The Mel38 score is positively correlated with increasing melanoma stage and is statistically significant independent to other variables.}

Mel38 scores for each specimen were calculated using support vector machine derived gene weights, as previously described. The scores range from 0 to 10 and are positivity correlated with the degree of malignancy, as shown by box plots of Mel38 vs AJCC stage (all samples) and vs MPath-Dx classes (primary lesions only), in Figure $3 \mathrm{~A}$ and $3 \mathrm{~B}$ respectively. The intraclass correlation of the score vs specimen status (naevi to metastatic melanoma) was 0.85 .

To verify that the Mel38 score is a continuous predictor of malignancy, independent to other clinicopathological variables, general linear models (GLM) were computed using patient age, gender, histological subtype, and AJCC Stage or MPATH-Dx class[9]. In both models, the genomic score was significantly different between AJCC stages and MPATH-Dx classes, independent to the other variables in the model $(P<0.001)$.

A separate multivariate analysis of Mel38 scores from invasive melanoma specimens only $(n=128)$ was performed, including, Breslow depth, tumour cell content, patient age, gender, and biopsy site. In this subset, the Mel38 score remained statistically significantly between disease stages $(P=0.012)$, independent of the other variables included in the statistical model, indicating robustness to other factors known to influence microRNA expression.

\section{The Mel38 score is a binary classifier of clinically higher- vs. lower-risk melanocytic lesions.}

Whilst the Mel38 score demonstrates a statistically significant continuous association with malignancy, inspection of the MPATH-Dx vs Mel38 box plot (Figure 3B) shows that the largest difference in genomic scores occurs between MPATH-Dx Class IV and V specimens. Notable differences in suggested clinical actions and patient outcome (i.e. 5- and 10-year disease specific survival rates) are also observed between these two classes, as summarised in Table 3. Further assessment of using the Mel38 score as a binary classification tool to assign higher-risk (M-PATH Dx Class V) versus lower-risk (Classes I-IV) lesions was then performed.

Receiver operator curve (ROC) analysis on Mel38 scores from 110 MPATH-Dx Class V vs. 175 Class I-IV specimens resulted in area under the curve (AUC) of 0.97 ( $95 \% \mathrm{Cl} 0.95$ to $0.99, \mathrm{P}<0.001)$ (Figure 4A). The binary fitted line plot shown in Figure 4B indicates the probability of MPATH-Dx Class V status according to a lesions Mel38 score.

Inspection of the ROC data and application of the Youden index revealed that a Mel38 score of $\geq 2.7$ was the optimal classification threshold for classifying a specimen as higher-risk; defined as an MPATH-Dx 
Class $\mathrm{V}$ or more advanced primary lesion[34]. This threshold corresponded to a true positive rate of $89 \%$ (i.e. Sensitivity; $95 \mathrm{Cl}: 82 \%$ to $94 \%$ ) and true negative rate of $94 \%$ (i.e. specificity; $\mathrm{Cl}$ : $89 \%$ to $97 \%$ ). These data suggest that using Mel38 with a threshold of $\geq 2.7$ to identify Class V / higher risk lesions would result in an underdiagnosis rate of $8.4 \%$, compared to the reported rate of $27 \%$ for conventional pathology alone[11].

ROC analysis was also performed for Mel38 scores and AJCC staging. An AUC of 0.96 (95\% Cl: 0.95 to 0.99). was computed, as shown in Figure $4 \mathrm{C}$. A binary fitted line plot was generated to visualise the probability of $\mathrm{a} \geq$ stage $1 \mathrm{~b}$ lesion versus Mel38 (Figure 4D).

\section{Mel38 profiling of Spitz naevi shows similarity to both benign and invasive melanoma.}

Spitz naevi are an uncommon type of melanocytic naevus, often histologically similar to melanoma and regarded as a challenging subcategory of melanocytic skin lesions to diagnose. Mel38-FFPE analysis was performed on twenty specimens of Spitz naevi submitted for routine histopathologic analysis from individuals ranging from two to thirty years old. As shown in Figure 1, the expression profile of the spitz naevi exhibits similarity to both benign and malignant lesions. Inspection of individual microRNAs in this figure reveals several that appear to have spitz-specific patterns of expression, i.e., hsa-mir-424-5p, hsamir-301a-3p and hsa-mir-1537-3p.

One way ANOVA of the Mel38 scores in spitz naevi versus other naevi subtypes revealed statistically significance $(P<0.001)$, with mean scores of 2.7 (95\% Cl: 2.5 to 3.0$)$ vs 1.5 ( $95 \% \mathrm{Cl}: 1.4$ to 1.7$)$, respectively, and significant pairwise differences to all naevi subtypes present. When analysed using a multivariate GLM, incorporating patient age, gender, and naevi subtype, the Mel38 scores of the spitz naevi were only significantly higher than the compound naevi subtype $(P=0.015)$. This suggests that the younger age of the spitz naevi patients compared to the rest of the cohort may be an influence on the Mel38 expression profile.

\section{The Mel38 score exhibits low technical variability between replicates and in longitudinal control sample analysis.}

RNA from 30 naevi and 30 melanoma samples was pooled, aliquoted into 3 ul volumes and analysed over a period of 7 (non-sequential) weeks. The Mel38 scores of each pool are shown longitudinally in Figure 5A. The mean score for the naevi control pool is 2.1 (standard deviation: 0.25 ) and 6.5 (standard deviation: 0.15 ) for the invasive melanoma pool. Additional control sample data will be generated over time and monitored to assess technical noise using Levey Jennings plots.

To assess the reproducibility of the genomic score throughout its dynamic range (i.e., 0 to 10), RNA from 89 samples was re-analysed and compared to the original Mel38 score. As shown in Figure 5B, there is 
high consistency between replicate scores, with an intraclass correlation coefficient of $0.96,(95 \% \mathrm{Cl} 0.94$ to 0.98 ) and no deviation from linearity (Cusum test, $P=0.62$ ). These results demonstrate that the Mel38 signature exhibits a high degree of longitudinal stability and technical reproducibility.

\section{Discussion}

Melanoma is a heterogeneous, progressive disease. It originates from melanocytic skin lesions and progresses through a series of well-documented stages of malignancy. In this study we describe the performance of a novel genomic biomarker for melanoma in a different specimen type (FFPE biopsy tissue) from that in which it was discovered (i.e. plasma). To our knowledge, this is the first study to demonstrate that a circulating series of microRNAs also exhibit disease-specific levels of expression in solid tissue. By comparing the 38-microRNA signature to relevant clinicopathological and specimen variables, its potential to contribute both accurate and reproducible information to a patient's diagnostic picture is shown.

The cohort presented in this study was designed to reflect the clinical continuum of melanoma and allowed the microRNA expression data generated to be analysed in relation to a spectrum of disease, from benign naevi to metastatic melanoma, as well as a binary condition, by classifying specimens into MPATH-Dx classes. In the former context, the Mel38 score exhibits a strong positive correlation with the progressive stages of melanoma, as represented by the ICC coefficient (0.85). When analysed as binary condition, with groups formed based on predetermined diagnostic classes with substantially different recommended follow-up actions and associated patient outcomes, a Mel38 threshold of $\geq 2.7$ was able to identify higher risk melanoma, equivalent to MPATH-Dx class $V$ or higher, with robust sensitivity and specificity.

A selection of spitz naevi specimens were included in this study due to their histological similarity with melanoma. Despite the fact they are usually diagnosed in younger patients, misdiagnosis of melanoma as a spitz nevus is an ongoing challenge[14]. Hierarchical clustering showed the expression profile of this subtype to have similarities to both benign and malignant disease, which was also reflected by their Mel38 scores. To date very few genomic studies of spitz naevi have been performed; only 56 out of 47,333 (0.1\%) melanocytic lesion profiles in the NCBI Gene Expression Omnibus are of spitzoid type[35]. This observation, albeit based on a limited sample size, may indicate there are a subset of spitz naevi which may benefit from additional treatment or monitoring.

Technical reproducibility of Mel38 signature was demonstrated by the low level of variation observed for both a melanoma and naevi control RNA pool, tested repeatedly over several months. Low variation and strong linearity were observed between a series of samples covering the full dynamic range of the score which were analysed in duplicate. These technical data are in line with those of other well validated genomic signatures used for individual patient management. Finally, the demonstrated reproducibility of the Mel38 score analyses exceeds that of conventional pathology based diagnoses, where almost one 
third of specimens may receive a different MPATH-Dx class when re-reviewed by the same pathologist[11].

Limitations of this study is the small numbers of less-common melanoma and naevi subtypes, such as amelanotic melanoma. Future work will focus on specific subtypes of melanoma and non-cancerous skin lesions which present challenges to accurately diagnose using conventional methods. A further limitation is not directly associating the genomic score to patient outcome or BRAF/NRAS status, due to the lack access to the molecular and clinical variables necessary for these types of analyses. However, in a previous in-silco study of previously published melanoma mRNA expression data, a significant association between MAPK pathway genes, melanoma-specific survival and the Mel38 signature was described[26].

It is important to highlight the proposed use of Mel38 is as a complementary molecular biomarker to conventional histopathology and is not intended to replace current diagnostic practices. Genomic testing, microscopic and immunohistochemical assessments of cellular/tissue structure all add unique and sometimes overlapping data points that contribute to a complete diagnostic picture. Incorporating genomic profiling into diagnostic workups may be useful for understanding future clinical events, including treatment reactions, that are inconsistent with the original diagnosis and stage of disease. Combining diagnostic modalities is becoming standard of care for many cancer types including breast (eg. Oncotype DX, MammaPrint, EndoPredict) and prostate (Oncotype DX, Prolaris, ProstaVysion), both of which have seen promising reductions in mortality over recent years, in part due to increased diagnostic precision and molecular disease subtyping. Clinical adaptation of Mel38-FFPE has the potential to add novel, robust and personalised genomic information to the diagnostic picture of patients with clinically suspicious melanocytic lesions.

\section{Conclusion}

Successful treatment of melanoma begins with accurate evaluation of melanocytic skin lesions. The consequences of over-or under-diagnosis could be avoided by using complementary diagnostic techniques. The Mel38 genomic signature, originally discovered in plasma and now validated in solid tissue, demonstrates a high degree of clinical accuracy and technical reproducibility. These factors make it suitable for use as an adjunct diagnostic biomarker in the clinical setting. By providing physicians with the opportunity to offer patients a genomic 'second opinion' of an excised lesion, it is hoped that diagnostic precision and accuracy can be improved and a greater understanding of genotype/phenotype differences in melanoma can be gained.

\section{Abbreviations}

- Mel38: 38 microRNA signature.

- GLM: General linear model.

- FFPE: Formalin fixed paraffin embedded. 
- Cl: Confidence Interval

- ROC: Receiver Operator Curve

- AUC: Area Under the Curve

- AJCC American Joint Committee on Cancer

\section{Declarations}

\section{Source of funding:}

Institutional funding.

\section{Disclosures:}

\section{Author abbreviations}

Ryan Van Laar: RVL, Samuel King: SK, Amicel Baynosa: AB, Richard McCoy: RM, Mirette Saad: MS, Sian Fereday: SF, Ingrid Winship: IW, Catherine Uzzell: CU, Anthony Landgren: AL.

\section{Ethics approval and consent to participate.}

The study was approved by Australian Clinical Labs internal medical advisory board and satisfies criteria for use of human tissue by diagnostic pathology companies as outlined by the Australian Government's National Health and Medical Research Council.

\section{Consent for publication}

N/A

\section{Availability of data and materials}

Data available upon request

\section{Declaration of interest}

RVL: Employee and shareholder in Geneseq Biosciences.

IW: Board Member and Shareholder in Geneseq Biosciences

SF: Consultant to Geneseq Biosciences

SK, RM, MS, CU, AL: Employee of Australian Clinical Labs

\section{Funding}




\section{Authors' contributions}

Study design and management: RVL, MS, IW, CU, AL

Laboratory work: SK, AM, RM

Pathology review: CU, AL

Data analysis: RVL, SF

Manuscript drafting and review: All.

\section{Acknowledgements}

The authors acknowledge the assistance of Amicel Baynosa and Keith Byron in the specimen processing and study initiation.

\section{References}

[1] N. H. Matthews, W. Q. Li, A. A. Qureshi, M. A. Weinstock, and E. Cho, "Epidemiology of Melanoma," in Cutaneous Melanoma: Etiology and Therapy, W. H. Ward and J. M. Farma Eds. Brisbane (AU), 2017.

[2] M. P. Staples, M. Elwood, R. C. Burton, J. L. Williams, R. Marks, and G. G. Giles, "Non-melanoma skin cancer in Australia: the 2002 national survey and trends since 1985," (in eng), Med J Aust, vol. 184, no. 1, pp. 6-10, Jan 2006, doi: 10.5694/j.1326-5377.2006.tb00086.x.

[3] H. G. Welch, B. L. Mazer, and A. S. Adamson, "The Rapid Rise in Cutaneous Melanoma Diagnoses," (in eng), N Engl J Med, vol. 384, no. 1, pp. 72-79, Jan 2021, doi: 10.1056/NEJMsb2019760.

[4] H. Kutzner et al., "Overdiagnosis of melanoma - causes, consequences and solutions," (in eng), $J$ Dtsch Dermatol Ges, vol. 18, no. 11, pp. 1236-1243, Nov 2020, doi: 10.1111/ddg.14233.

[5] R. Rubin, "Melanoma Diagnoses Rise While Mortality Stays Fairly Flat, Raising Concerns About Overdiagnosis," (in eng), JAMA, vol. 323, no. 15, pp. 1429-1430, Apr 2020, doi: 10.1001/jama.2020.2669.

[6] K. L. Nufer, A. P. Raphael, and H. P. Soyer, "Dermoscopy and Overdiagnosis of Melanoma In Situ," (in eng), JAMA Dermatol, vol. 154, no. 4, pp. 398-399, 04 2018, doi: 10.1001/jamadermatol.2017.6448.

[7] K. Seiger, C. D. Schmults, A. W. Silk, and E. S. Ruiz, "Cost and utilization of immunotherapy and targeted therapy for melanoma: Cross-sectional analysis in the Medicare population, 2013 and 2015," (in eng), J Am Acad Dermatol, vol. 82, no. 3, pp. 761-764, 03 2020, doi: 10.1016/j.jaad.2019.10.023.

[8] J. Berk-Krauss, J. A. Stein, J. Weber, D. Polsky, and A. C. Geller, "New Systematic Therapies and Trends in Cutaneous Melanoma Deaths Among US Whites, 1986-2016," (in eng), Am J Public Health, vol. 110, no. 5, pp. 731-733, 05 2020, doi: 10.2105/AJPH.2020.305567. 
[9] M. W. Piepkorn et al., "The MPATH-Dx reporting schema for melanocytic proliferations and melanoma," (in eng), J Am Acad Dermatol, vol. 70, no. 1, pp. 131-41, Jan 2014, doi:

10.1016/j.jaad.2013.07.027.

[10] A. C. Radick et al., "Terminology for Melanocytic Skin Lesions and the MPATH-Dx Classification Schema: A Survey of Dermatopathologists," (in eng), J Cutan Pathol, Sep 2020, doi: 10.1111/cup.13873.

[11] J. G. Elmore et al., "Pathologists' diagnosis of invasive melanoma and melanocytic proliferations: observer accuracy and reproducibility study," BMJ, 10.1136/bmj.j2813 vol. 357, 2017.

[12] S. Ronen et al., "Discordance in Diagnosis of Melanocytic Lesions and Its Impact on Clinical Management," (in eng), Arch Pathol Lab Med, Feb 2021, doi: 10.5858/arpa.2020-0620-OA.

[13] S. Patrawala et al., "Discordance of histopathologic parameters in cutaneous melanoma: Clinical implications," (in eng), J Am Acad Dermatol, vol. 74, no. 1, pp. 75-80, Jan 2016, doi:

10.1016/j.jaad.2015.09.008.

[14] P. Gerami et al., "Histomorphologic assessment and interobserver diagnostic reproducibility of atypical spitzoid melanocytic neoplasms with long-term follow-up," (in eng), Am J Surg Pathol, vol. 38, no. 7, pp. 934-40, Jul 2014, doi: 10.1097/PAS.0000000000000198.

[15] H. M. Rolfe, "Accuracy in skin cancer diagnosis: a retrospective study of an Australian public hospital dermatology department," (in eng), Australas J Dermatol, vol. 53, no. 2, pp. 112-7, May 2012, doi: 10.1111/j.1440-0960.2011.00855.x.

[16] R. L. Wilson, B. A. Yentzer, S. P. Isom, S. R. Feldman, and A. B. Fleischer, "How good are US dermatologists at discriminating skin cancers? A number-needed-to-treat analysis," (in eng), J Dermatolog Treat, vol. 23, no. 1, pp. 65-9, Feb 2012, doi: 10.3109/09546634.2010.512951.

[17] C. Rosendahl et al., "Measuring performance in skin cancer practice: the SCARD initiative," Int J Dermatol, vol. 50, no. 1, pp. 44-51, Jan 2011, doi: 10.1111/j.1365-4632.2010.04608.x.

[18] R. Corona et al., "Interobserver variability on the histopathologic diagnosis of cutaneous melanoma and other pigmented skin lesions," (in eng), J Clin Oncol, vol. 14, no. 4, pp. 1218-23, Apr 1996, doi: 10.1200/JCO.1996.14.4.1218.

[19] U. S. P. S. T. Force et al., "Screening for Skin Cancer: US Preventive Services Task Force Recommendation Statement," JAMA, vol. 316, no. 4, pp. 429-35, Jul 26 2016, doi: 10.1001/jama.2016.8465.

[20] E. B. Hawryluk et al., "Histologically challenging melanocytic tumors referred to a tertiary care pigmented lesion clinic," (in eng), J Am Acad Dermatol, vol. 67, no. 4, pp. 727-35, Oct 2012, doi: 10.1016/j.jaad.2012.02.036. 
[21] B. A. Shoo, R. W. Sagebiel, and M. Kashani-Sabet, "Discordance in the histopathologic diagnosis of melanoma at a melanoma referral center," (in eng), J Am Acad Dermatol, vol. 62, no. 5, pp. 751-6, May 2010, doi: 10.1016/j.jaad.2009.09.043.

[22] K. Sundarbose, R. V. Kartha, and S. Subramanian, "MicroRNAs as Biomarkers in Cancer," (in eng), Diagnostics (Basel), vol. 3, no. 1, pp. 84-104, Jan 16 2013, doi: 10.3390/diagnostics3010084.

[23] !!! INVALID CITATION !!! [23, 24].

[24] R. K. van Laar, M. T. Lincoln, and B. J. van Laar, "A plasma microRNA biomarker of melanoma as a personalised assessment of treatment response," (in eng), Melanoma Res, vol. 29, no. 1, pp. 19-22, Feb 2019, doi: 10.1097/CMR.0000000000000492.

[25] R. Van Laar, M. Lincoln, and B. Van Laar, "Development and validation of a plasma-based melanoma biomarker suitable for clinical use," Br J Cancer, vol. 118, no. 6, pp. 857-866, Mar 20 2018, doi: 10.1038/bjc.2017.477.

[26] R. Van Laar, M. Lincoln, and S. Fereday, "Characterisation and validation of Mel38; A multi-tissue microRNA signature of cutaneous melanoma," PLoS One, vol. 14, no. 2, p. e0211504, 2019, doi: 10.1371/journal.pone.0211504.

[27] R. J. Sullivan and K. Flaherty, "MAP kinase signaling and inhibition in melanoma," (in eng), Oncogene, vol. 32, no. 19, pp. 2373-9, May 2013, doi: 10.1038/onc.2012.345.

[28] J. E. Gershenwald et al., "Melanoma staging: Evidence-based changes in the American Joint Committee on Cancer eighth edition cancer staging manual," (in eng), CA Cancer J Clin, vol. 67, no. 6, pp. 472-492, Nov 2017, doi: 10.3322/caac.21409.

[29] P. Sainsbury, "Development and oversight of ethical health promotion quality assurance and evaluation activities involving human participants," (in eng), Health Promot J Austr, vol. 26, no. 3, pp. 176181, Dec 2015, doi: 10.1071/HE15045.

[30] K. Hajian-Tilaki, "Sample size estimation in diagnostic test studies of biomedical informatics," (in eng), J Biomed Inform, vol. 48, pp. 193-204, Apr 2014, doi: 10.1016/j.jbi.2014.02.013.

[31] D. Waggott, K. Chu, S. Yin, B. G. Wouters, F. F. Liu, and P. C. Boutros, "NanoStringNorm: an extensible R package for the pre-processing of NanoString mRNA and miRNA data," Bioinformatics, vol. 28, no. 11, pp. 1546-8, Jun 1 2012, doi: 10.1093/bioinformatics/bts188.

[32] F. Schoonjans, A. Zalata, C. E. Depuydt, and F. H. Comhaire, "MedCalc: a new computer program for medical statistics," (in eng), Comput Methods Programs Biomed, vol. 48, no. 3, pp. 257-62, Dec 1995.

[33] R: A language and environment for statistical computing. R Foundation for Statistical Computing, 2010. 

doi: 10.1002/1097-0142(1950)3:1<32::aid-cncr2820030106>3.0.co;2-3.

[35] R. Edgar, M. Domrachev, and A. E. Lash, "Gene Expression Omnibus: NCBI gene expression and hybridization array data repository," Nucleic Acids Res, vol. 30, no. 1, pp. 207-10, Jan 12002.

[36] J. O. Westgard, P. L. Barry, M. R. Hunt, and T. Groth, "A multi-rule Shewhart chart for quality control in clinical chemistry," Clin Chem, vol. 27, no. 3, pp. 493-501, Mar 1981.

[37] M. G. Niebling, L. E. Haydu, R. Z. Karim, J. F. Thompson, and R. A. Scolyer, "Pathology review significantly affects diagnosis and treatment of melanoma patients: an analysis of 5011 patients treated at a melanoma treatment center," (in eng), Ann Surg Oncol, vol. 21, no. 7, pp. 2245-51, Jul 2014, doi: 10.1245/s10434-014-3682-x.

\section{Tables}

Table 1: Summary of melanoma histopathology studies reporting diagnostic accuracy

\begin{tabular}{|l|c|c|c|c|}
\hline Study of melanoma histopathology accuracy: & $\begin{array}{c}\text { Year } \\
\text { of } \\
\text { study }\end{array}$ & $\begin{array}{c}\text { Number } \\
\text { of cases }\end{array}$ & $\begin{array}{c}\text { Rate of major } \\
\text { /minor } \\
\text { diagnostic } \\
\text { discordance }\end{array}$ & $\begin{array}{c}\text { Estimated } \\
\text { rate of } \\
\text { change in } \\
\text { management }\end{array}$ \\
\hline $\begin{array}{l}\text { Discordance in Diagnosis of Melanocytic Lesions and Its Impact } \\
\text { on Clinical Management: A Melanoma Referral Center } \\
\text { Experience With 1521 Cases.[12] }\end{array}$ & 2021 & 1,521 & $20 \% / 49 \%$ & $11 \%$ \\
\hline $\begin{array}{l}\text { Pathologists diagnosis of invasive melanoma and melanocytic } \\
\text { proliferations: observer accuracy and reproducibility study.[11] }\end{array}$ & 2017 & 240 & $17 \% / 48 \%$ & $17 \%$ \\
\hline $\begin{array}{l}\text { Discordance of histopathologic parameters in cutaneous } \\
\text { melanoma: Clinical implications.[13] }\end{array}$ & 2016 & 588 & $19 \% /$ NA & $18 \%$ \\
\hline $\begin{array}{l}\text { Pathology review significantly affects diagnosis and treatment of } \\
\text { melanoma patients: an analysis of 5011 patients treated at a } \\
\text { melanoma treatment center.[37] }\end{array}$ & 5014 & $5 \% / 22 \%$ & $11 \%$ \\
\hline $\begin{array}{l}\text { Histomorphologic Assessment and Interobserver Diagnostic } \\
\text { Reproducibility of Atypical Spitzoid Melanocytic Neoplasms } \\
\text { With Long-term Follow-up[14] }\end{array}$ & 2014 & 75 & $70 \% /$ NA & N/A \\
\hline $\begin{array}{l}\text { Histologically challenging melanocytic tumors referred to a } \\
\text { tertiary care pigmented lesion clinic.[20] }\end{array}$ & 2012 & 478 & $35 \% /$ NA & $13 \%$ \\
\hline $\begin{array}{l}\text { Discordance in the histopathologic diagnosis of melanoma at a } \\
\text { melanoma referral center. [21] }\end{array}$ & 2010 & 392 & $14 \% /$ NA & $14 \%$ \\
\hline $\begin{array}{l}\text { Interobserver variability on the histopathologic diagnosis of } \\
\text { cutaneous melanoma and other pigmented skin lesions.[18] }\end{array}$ & 1996 & 140 & $39 \% /$ NA & $39 \%$ \\
\hline
\end{tabular}

Table 2: Patient and specimen demographics 


\begin{tabular}{|l|l|l|}
\hline Descriptor & Number & Percent \\
\hline Age: & & \\
Mean & 60 & \\
$<30$ & 19 & $7 \%$ \\
$31-40$ & 37 & $13 \%$ \\
$41-50$ & 34 & $12 \%$ \\
$51-60$ & 41 & $14 \%$ \\
$>60$ & 157 & $54 \%$ \\
\hline Gender & & \\
Male: & 154 & $53 \%$ \\
Female: & 134 & $47 \%$ \\
\hline Naevi & & \\
Intradermal & 16 & $17 \%$ \\
Compound & 37 & $40 \%$ \\
Junctional & 16 & $17 \%$ \\
Spitz & 20 & $22 \%$ \\
Other & 4 & $4 \%$ \\
\hline Melanoma in-situ & & \\
Lentigo maligna & 33 & $69 \%$ \\
Superficial spreading & 6 & $12 \%$ \\
Other & 9 & $19 \%$ \\
\hline Invasive melanoma & & \\
Superficial spreading & 84 & $50 \%$ \\
Nodular & 29 & $17 \%$ \\
Lentigo maligna & 8 & $5 \%$ \\
Other & 46 & $28 \%$ \\
\hline AJCC Clinical Stage: & & \\
IA & 58 & $35 \%$ \\
IB & 19 & $11 \%$ \\
IIA & 17 & $10 \%$ \\
IIB & 21 & $13 \%$ \\
IIC & 13 & $8 \%$ \\
III/IV & 39 & $23 \%$ \\
\hline MPATH-Dx Class & 33 & $11 \%$ \\
I & 44 & $15 \%$ \\
II & 50 & $17 \%$ \\
III & 54 & $18 \%$ \\
IV & 113 & $38 \%$ \\
V & \\
\hline
\end{tabular}

Table 3: MPATH-Dx classes, mean Mel38 scores and associated clinicopathological variables, including suggested clinical actions. *Melanoma specific survival figures based on AJCC melanoma staging and outcome data corresponding to the T-stage associated with each MPATH-Dx class. \# Suggested clinical actions assume representative sampling and positive margins. Reproduced from Piepkorn et al [9] 


\begin{tabular}{|c|c|c|c|c|c|}
\hline $\begin{array}{l}\text { MPATH-Dx } \\
\text { Class: }\end{array}$ & I & II & III & IV & V \\
\hline $\begin{array}{l}\text { Mel38 score } \\
\text { (mean) }\end{array}$ & 1.5 & 1.7 & 1.7 & 2.1 & 5.2 \\
\hline $\begin{array}{l}\text { Mel38 risk } \\
\text { group }\end{array}$ & \multicolumn{4}{|c|}{ Low risk } & High Risk \\
\hline T stage & $\mathrm{N} / \mathrm{A}$ & $\mathrm{N} / \mathrm{A}$ & 0 & $\mathrm{~T} 1 \mathrm{a}$ & $\mathrm{T} 1 \mathrm{~b}$ or more \\
\hline $\begin{array}{l}\text { Perceived risk } \\
\text { for progression }\end{array}$ & Very low risk & Low risk & Higher risk & $\begin{array}{c}\text { Substantial risk } \\
\text { for local/regional } \\
\text { progression }\end{array}$ & $\begin{array}{c}\text { Greatest risk for } \\
\text { regional and/or distant } \\
\text { metastases }\end{array}$ \\
\hline $\begin{array}{l}5 \text { /10-year } \\
\text { melanoma- } \\
\text { specific } \\
\text { survival* }\end{array}$ & $100 \% / 100 \%$ & $100 \% / 100 \%$ & $100 \% / 100 \%$ & $98 \% / 96 \%$ & $\leq 93 \% / \leq 89 \%$ \\
\hline $\begin{array}{l}\text { Suggested } \\
\text { clinical } \\
\text { action }^{\#} \text { : }\end{array}$ & $\begin{array}{l}\text { No further } \\
\text { treatment / } \\
\text { follow up as } \\
\text { required. }\end{array}$ & $\begin{array}{l}\text { Narrow but } \\
\text { complete } \\
\text { excision } \\
(<5 \mathrm{~mm})\end{array}$ & $\begin{array}{l}\text { Complete excision } \\
\text { with at least } 5 \mathrm{~mm} \text { but } \\
<10 \mathrm{~mm} \text { margins. }\end{array}$ & $\begin{array}{c}\text { Wide local } \\
\text { excision with } \\
\geq 10 \mathrm{~mm} \text { margins. }\end{array}$ & $\begin{array}{c}\text { Wide local excision } \\
\text { with } \geq 10 \mathrm{~mm} \text { margins. } \\
\text { Consideration of } \\
\text { sentinel lymph node } \\
\text { biopsy, adjuvant } \\
\text { therapy. }\end{array}$ \\
\hline $\begin{array}{l}\text { Clinical action } \\
\text { likely to } \\
\text { require } \\
\text { referral: }\end{array}$ & \multicolumn{4}{|c|}{ No } & Yes \\
\hline
\end{tabular}

\section{Figures}




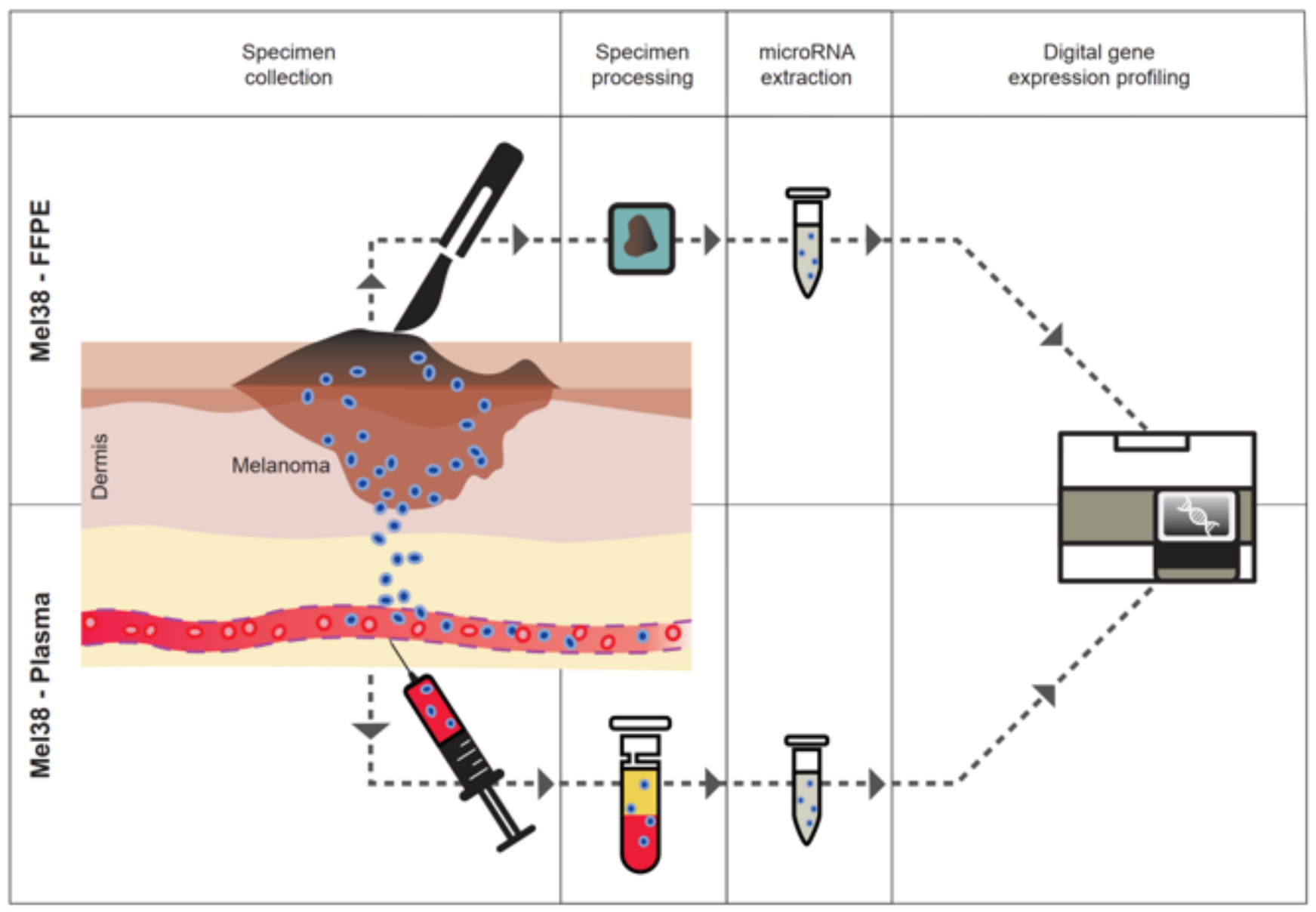

Figure 1

Schematic diagram of the development of Mel38, a microRNA signature of melanoma originally identified in plasma (lower section), and its adaptation to FFPE melanocytic skin lesions (upper section) using digital gene expression profiling methods and classification algorithms previously described. 


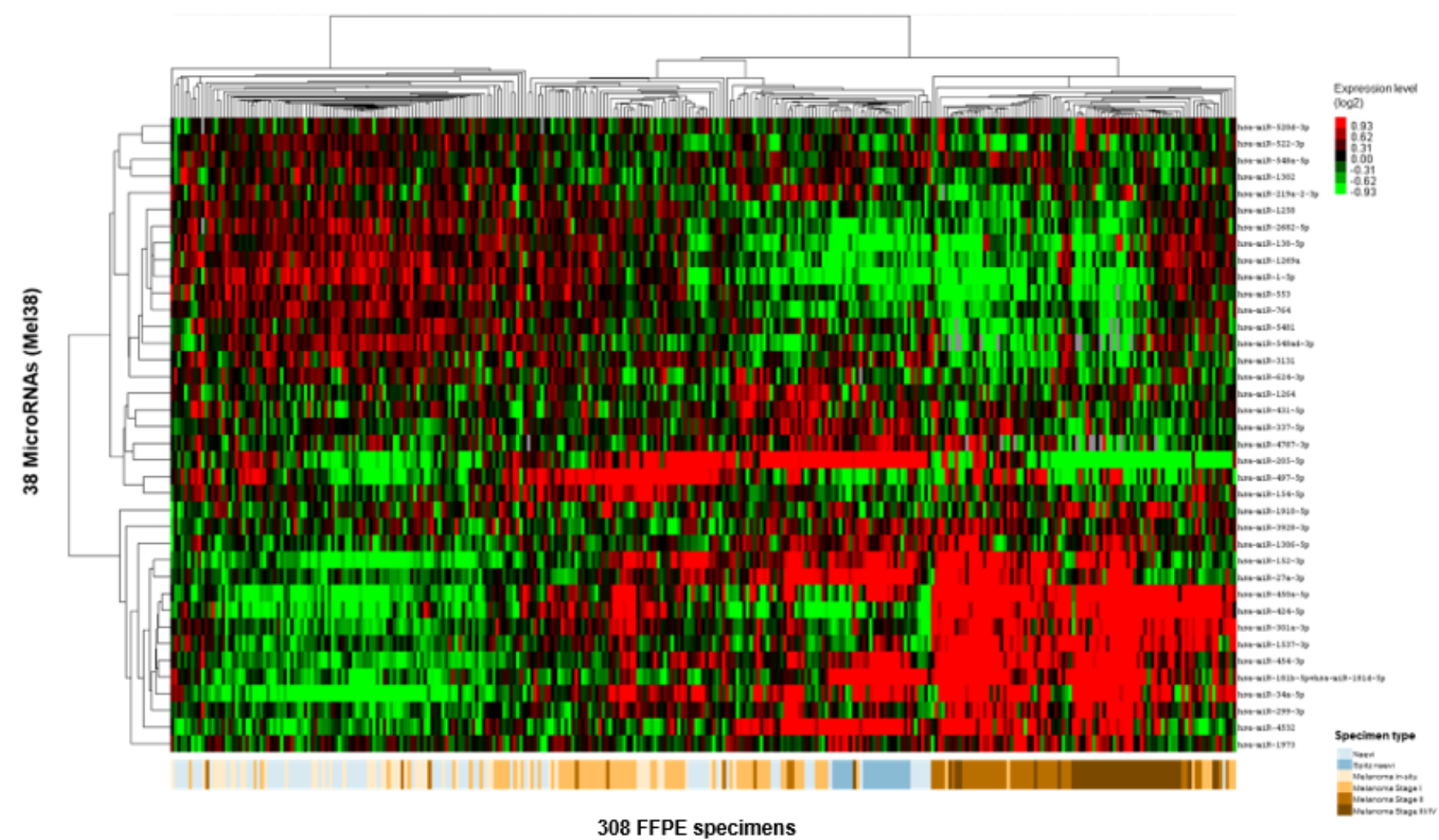

\section{Figure 2}

Supervised hierarchical clustering of the Mel38 signature data from 308 FFPE specimens of benign naevi, melanoma in-situ and invasive melanoma. The clustering pattern revealed shows blocks of up and down regulation, reflecting the raw data used by the Mel38-FFPE classifier to compute each patient's individual Mel38-FFPE score.

A.

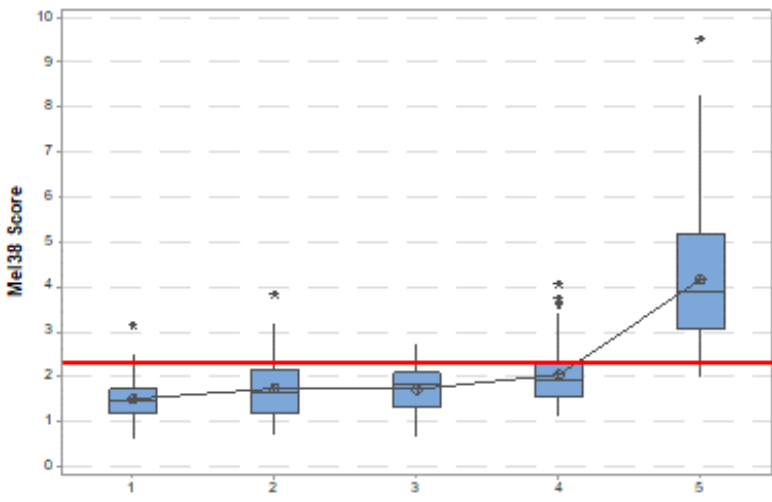

MPATH-Dx Class

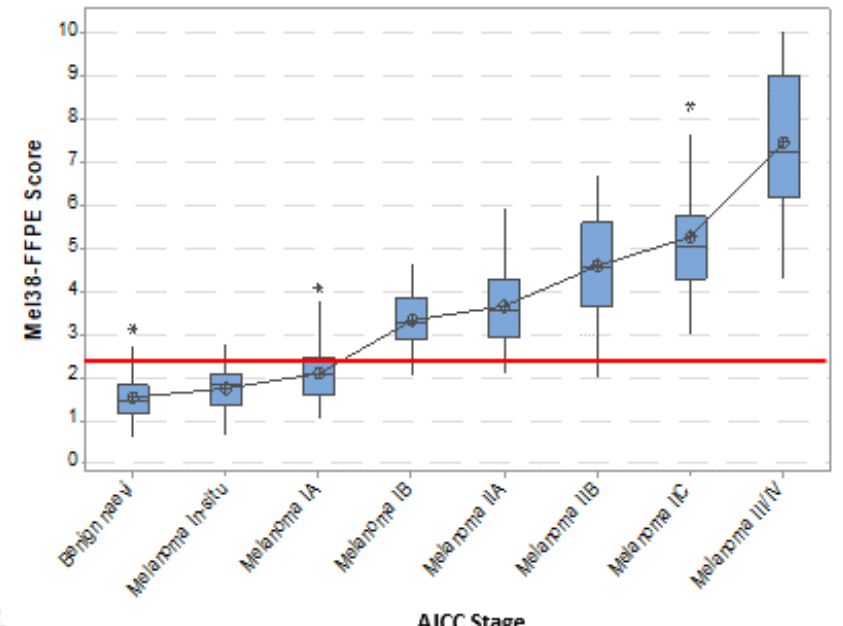

$B$.

AJCC Stage

\section{Figure 3}

(A) Box plot of Mel38-FFPE scores of primary melanocytic lesions by MPATH-Dx class. (B) Box plot of Mel38-FFPE scores of primary melanocytic lesions by AJCC clinical stage (8th ed). Connecting lines 
between boxes correspond to mean values. Red horizonal line indicates the Mel38 classification threshold of 2.7 for higher-risk lesions.

A. Mel38-FFPE Stratification of MPATH-Dx Class I-IV vs. 2V lesions

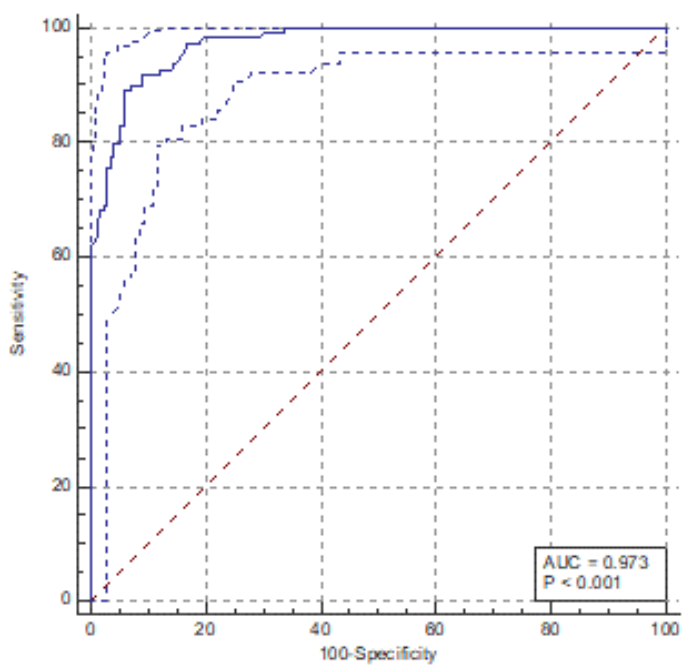

C. Mel38-FFPE Stratification of AJCC Stage $\leq 1 \mathrm{~A}$ v.s $21 B$ lesions

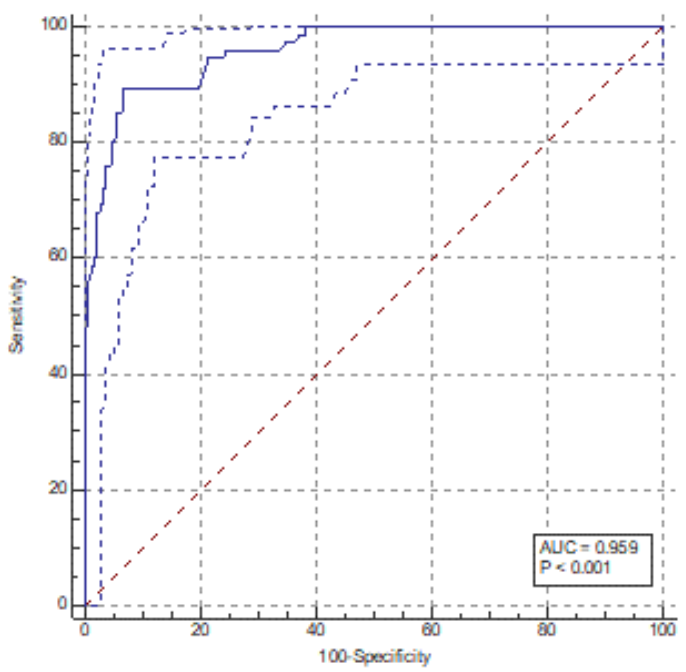

B. Probability of z MPATH Class-V status vs Mel38-FFPE score

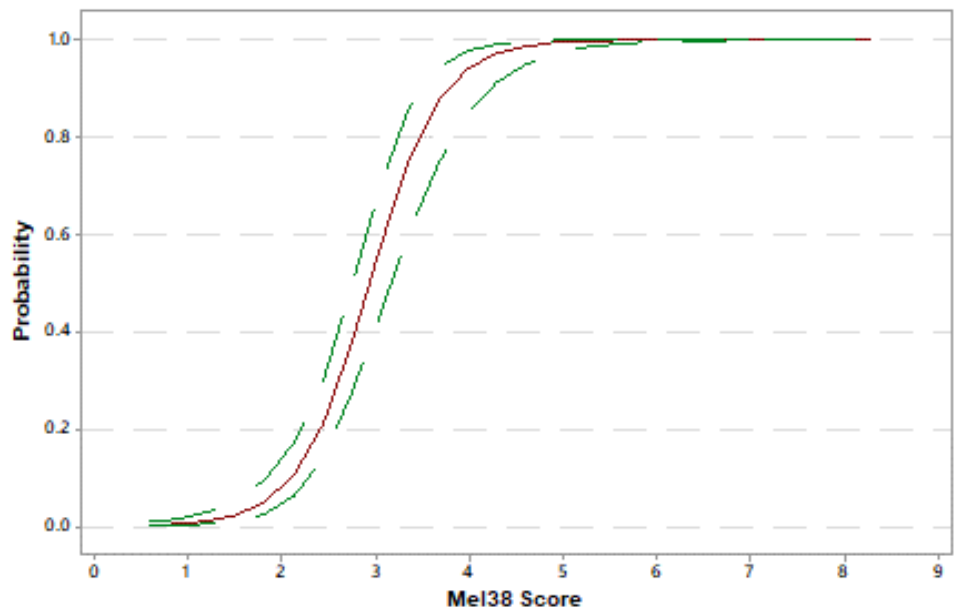

D. Probability of $\geq$ Stage $1 B$ status vs. Mel38-FFPE score

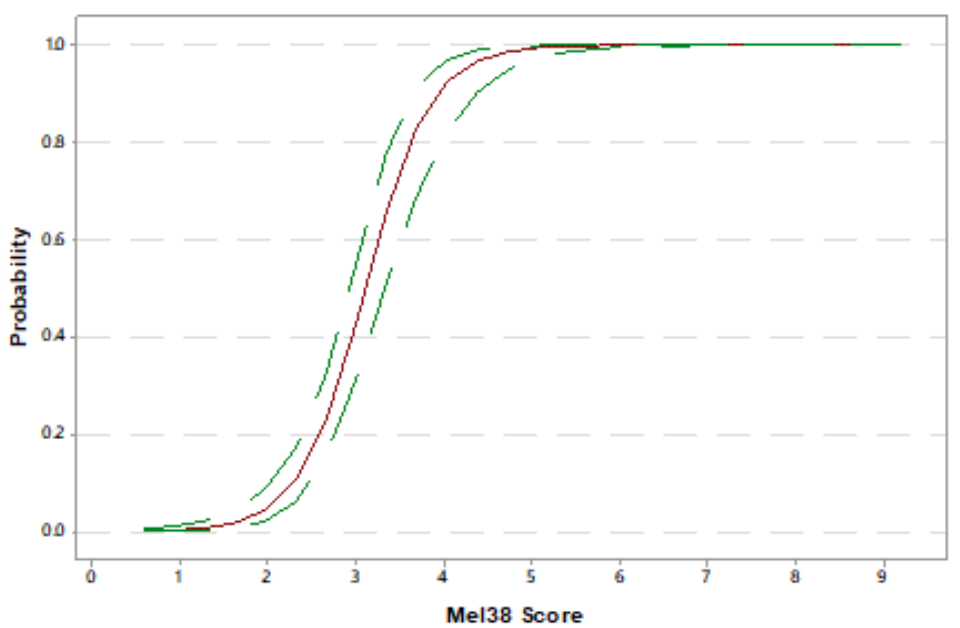

\section{Figure 4}

(A) Receiver operator curve analysis of Mel38 scores from primary melanocytic skin lesions vs. MPATHDx Classes I- IV vs. Class V. AUC: $0.97,95 \% \mathrm{Cl}$ : 0.95 to 0.99. (B) Binary fitted line plot from logistic regression analysis of Mel38 scores vs probability of MPATH-Dx Class V melanoma. (C) Receiver operator curve analysis of Mel38 scores from primary melanocytic skin lesions vs. AJCC Stage $\leq 1 \mathrm{~A}$ vs $\geq 1$ B. AUC: $0.96,95 \% \mathrm{Cl}: 0.95$ to 0.99 . (B) Binary fitted line plot from logistic regression analysis of Mel38 scores vs probability of MPATH-Dx Class V melanoma. 
A.

Pooled Melanoma RNA control:

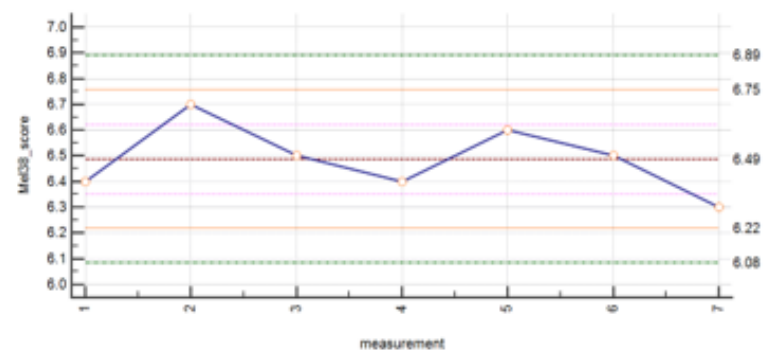

Pooled Noevi RNA control:

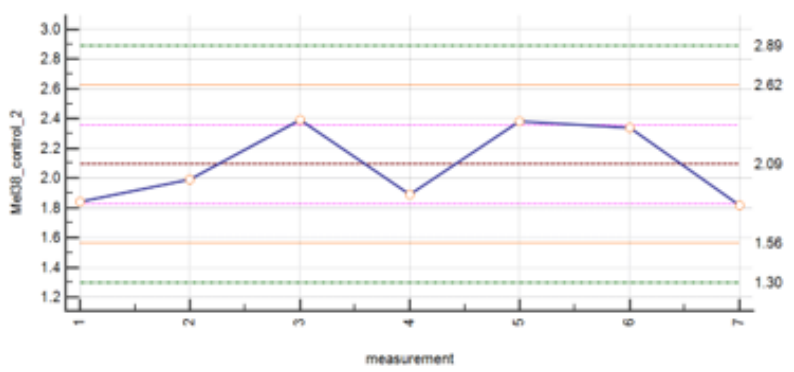

B.

Replicate analysis of individual potient specimens:

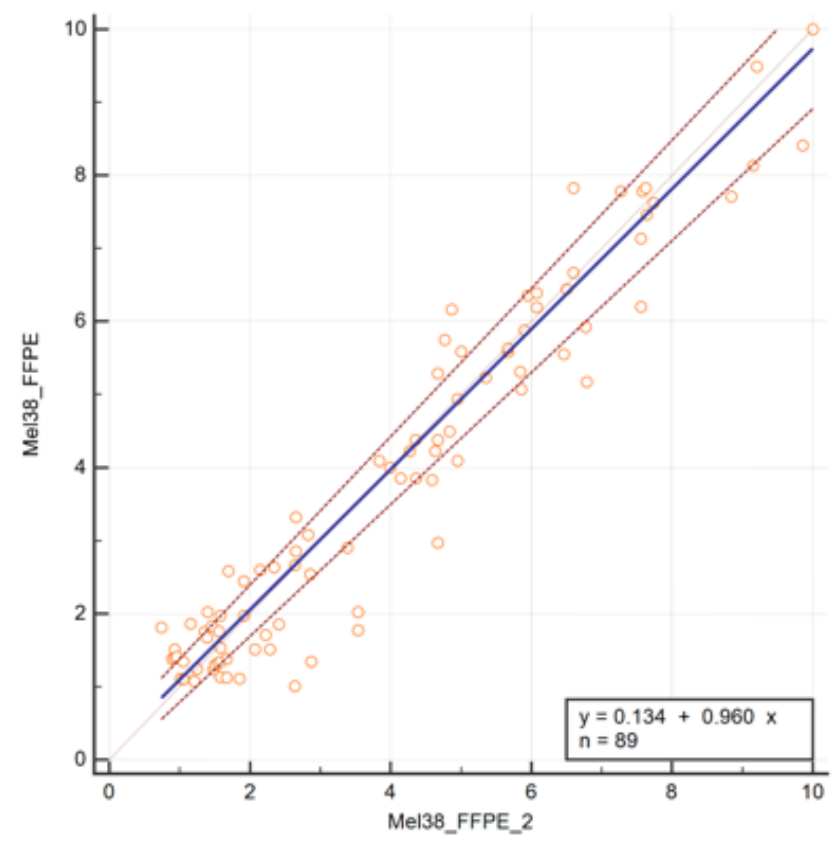

\section{Figure 5}

(A) Longitudinal analysis of control sample Mel38 scores. Dashed horizontal lines corresponding to 1s, $2 s$ and $3 s$ Westguard rules indicated[36]. (B) Passing and Bablok regression of replicate Mel38-FFPE specimens. No significant deviation from linearity is detected (Cusom test $P=0.62$ ) and intraclass correlation of 0.96 ( 0.94 to 0.98 ) reflects the signatures high degree of reproducibility.

\section{Supplementary Files}

This is a list of supplementary files associated with this preprint. Click to download.

- SupplementaryInformation.docx 